\title{
Serological responses in porcine cysticercosis: A link with the parasitological outcome of infection
}

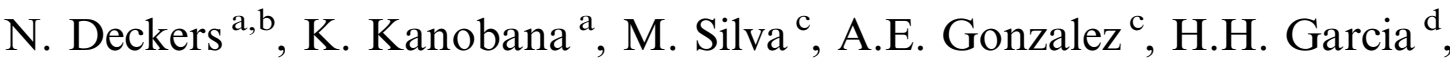 \\ R.H. Gilman ${ }^{\text {e,f }}$, P. Dorny ${ }^{\mathrm{a}, \mathrm{b}, *}$ \\ ${ }^{a}$ Department of Animal Health, Institute of Tropical Medicine, Nationalestraat 155, B-2000 Antwerp, Belgium \\ ${ }^{\mathrm{b}}$ Department of Virology, Parasitology and Immunology, Faculty of Veterinary Medicine, Ghent University, Belgium \\ ${ }^{\mathrm{c}}$ Facultad de Medicina Veterinaria, Universidad Nacional Mayor de San Marcos, Lima, Peru \\ ${ }^{\mathrm{d}}$ Cysticercosis Unit, Instituto de Ciencias Neurologicas, Lima, Peru \\ ${ }^{\mathrm{e}}$ Bloomberg School of Public Health. Johns Hopkins University, Baltimore, USA \\ ${ }^{\mathrm{f}}$ Department of Microbiology, Universidad Peruana Cayetano Heredia, Lima, Peru
}

Received 15 November 2007; received in revised form 8 January 2008; accepted 15 January 2008

\begin{abstract}
An oral infection model with Taenia solium whole proglottids was used to study host-parasite relationships and the mechanisms underlying resistance to infection in pigs. In addition, an attempt was made to link the parasitological findings to serological data. Groups of six piglets aged 1, 3 and 5 months were infected and slaughtered 3 months p.i. Circulating antibody and antigen levels were monitored weekly. At autopsy total cyst counts were performed. Although the detailed carcass dissection at necropsy revealed a high variation in the number of cysts, the trend was that the number of viable cysts decreased with the age at which the animals were infected. The kinetics of the antigen levels throughout the course of the infection differed markedly between the three age groups of the experimental infection model. In the younger animals, a fast increase in titres of circulating antigen was observed in most animals, reaching a plateau as early as 2 weeks p.i. Besides its faster increase, antigen levels in pigs infected at younger ages also reached higher levels than in older animals and were associated with weaker antibody responses. Results also demonstrated that a relationship exists between the number of cysts and the titre of circulating antigen. This is promising in view of the development of an assay to quantify the progress of an active T. solium infection and would be a useful tool in epidemiological studies to assess the infection burden and the risk of transmission of the disease. The use of specific antibody-detection assays combined with circulating antigen detection could improve our understanding of this relationship.
\end{abstract}

(C) 2008 Australian Society for Parasitology Inc. Published by Elsevier Ltd. All rights reserved.

Keywords: Taenia solium; Pig; Experimental infection; Antibodies; Circulating antigen

\section{Introduction}

Porcine and human cysticercosis caused by Taenia solium are major socio-economic concerns in developing countries where hygiene conditions are poor and pig access to human faeces is facilitated (Garcia et al., 1993). In pigs, the disease is characterised by the development of the larval

\footnotetext{
${ }^{*}$ Corresponding author. Address: Department of Animal Health, Institute of Tropical Medicine, Nationalestraat 155, B-2000 Antwerp, Belgium. Tel.: +32 3247 6394; fax: +32 32476268 .

E-mail address: pdorny@itg.be (P. Dorny).
}

stage (cysticerci) in tissues, mostly without clinical symptoms. Human cysticercosis occurs in the skeletal musculature, the CNS (neurocysticercosis (NCC)) or the eye (Garcia et al., 2002). NCC is endemic and a main cause of late-onset epilepsy in most developing countries (Schantz et al., 1994; Garcia et al., 1997). Due to immigration from endemic areas it is also increasingly common in industrialised countries (Schantz et al. 1998; Garcia and Del Bruto, 2000).

Transmission of the disease can be interrupted by increased hygienic measures which are, however, difficult 
to implement in developing countries. Therefore, alternative measures for reduction of transmission are being developed, such as health education, mass chemotherapy in humans and pigs, and vaccination of pigs (Sarti et al., 1997; Garcia et al., 2007). Infected pigs treated with a single-dose of $30 \mathrm{mg} / \mathrm{kg}$ of oxfendazole became refractory to re-infection 3 months later (Gonzalez et al., 1996, 1997, 1998). Another approach initiated by different research groups is vaccination of pigs aiming at interrupting the parasite's life-cycle by preventing pigs from acquiring the larval stage (Nascimento et al., 1995; Sciutto et al., 1995; Flisser and Lightowlers, 2001; Flisser et al., 2004; Gonzalez et al., 2005). A synthetic peptide-based vaccine has also proved to have therapeutic activity (de Aluja et al., 2005).

Interventions based on immunisation of pigs are not yet commonly applied. This is partly due to a lack of understanding of the host-parasite interactions and the immune mechanisms involved in resistance to the disease. Prerequisites to evaluate results of immunisation are (i) to understand whether all pigs equally acquire the disease upon infection and (ii) to determine how long metacestodes remain alive without intervention.

Thus, reproducible and standardised experimental infections with $T$. solium are needed, both for vaccination studies and to learn more about the course and progress of infection. Recently the cysticercosis working group in Peru succeeded in developing a reproducible experimental model in pigs in which pigs are orally infected with a whole proglottid embedded in a banana ball. The advantage of oral infection is that it mimics the natural mode of infection. The use of a complete proglottid rather than eggs in solution has the disadvantage that the number of eggs ingested by the animals might differ. However, the infectivity of the eggs will also likely be more comparable with what occurs under natural conditions.

In this paper, we report on the serological response of experimentally infected pigs and an attempt was made to link the serological and the parasitological responses to infection. In a second part of the study, we extended this link by investigating whether a quantitative relationship could be established between the titre of circulating antigen in the serum of animals and the number of viable cysts at necropsy.

\section{Materials and methods}

\subsection{Animals and experimental design 1}

Eighteen piglets were purchased from a commercial cysticercosis-free farm in Lima, Peru and maintained at specific pathogen free facilities of the School of Veterinary Medicine of the San Marcos University (UNMSM) in Lima. Animals were housed individually and had free access to water and feed. The cysticercosis-free status of the animals was confirmed by analysis of pre-infection serum with the electro immuno-transfer blot (EITB) (Tsang et al., 1989). Six piglets were infected at the age of 1 month, six piglets at the age of 3 months, and six piglets at the age of 5 months.

Animals received a single infective dose of $T$. solium eggs. Briefly, following a 1-week period of becoming accustomed to eating banana and honey balls, all animals were infected with one gravid proglottid embedded in a banana and honey ball. Following ingestion of the banana ball, animals were observed for $2 \mathrm{~h}$ to ensure the infective dose was not regurgitated. Animals were bled weekly during the first month p.i. and every 2 weeks thereafter, until the end of the experiment. Animals were euthanised approximately 3 months p.i. A systematic dissection of the full carcass was then performed. The number of cysts was enumerated and viable and degenerated cysts were differentiated. Viable cysticerci had well-limited thin-walled cystic structures containing clear vesicular fluid and a visible whitish protoscolex. Degenerated cysticerci were non-cystic but rather had semi-solid caseous masses with no discernible parasite structures (Phiri et al., 2006). This experiment was reviewed and approved by the Committee on Animal research of the School of Veterinary Medicine, UNMSM.

\subsection{Animals and experimental design 2}

In a second part of the study, we aimed to investigate whether a quantitative relationship could be established between the total number of viable cysts found at necropsy and the level of circulating antigen in serum. For this purpose, serum samples from different experiments were used. All experiments were part of the research performed by the Department of Preventive Medicine of the School of Veterinary Medicine of the UNMSM and approved by the Committee on Animal research of the School of Veterinary Medicine, UNMSM.

The criteria for inclusion of the serum samples in the study were (i) the availability of sufficient material and (ii) the precise determination of the parasite burden (as well as differentiation between degenerated and viable cysts) by detailed carcass dissection following slaughter. This study included 98 porcine serum samples originating from 98 experimentally infected animals. The age of the animals varied between 3-12 months at slaughter.

\subsection{Detection of circulating antigen}

For experimental design 1, the monoclonal based sandwich ELISA for the detection of circulating antigen (Brandt et al., 1992; Dorny et al., 2000) was performed twice. First, the standard protocol was used as described previously (Dorny et al., 2000) with a few modifications: incubation steps were reduced from $1 \mathrm{~h}$ to $30 \mathrm{~min}$ (coating) or $15 \mathrm{~min}$ (other steps); all incubations were done on a shaking plate except for the last step (substrate); streptavidin-horseradish peroxydase (Jackson Immunoresearch lab Inc.) diluted $1 / 10,000$ was used as the conjugate. 
Table 1

Number and classification of cysts following necropsy of piglets infected with Taenia solium at the age of 1,3 or 5 months

\begin{tabular}{|c|c|c|c|c|}
\hline $\operatorname{Age}^{a}$ & Animal no. & Total no. of cysts & No. of viable cysts & No. of degenerated cysts \\
\hline \multirow[t]{8}{*}{1 month } & 8115 & 6246 & 6210 & 36 \\
\hline & 8116 & 3199 & 3295 & 4 \\
\hline & 8113 & 2038 & 1946 & 92 \\
\hline & 8114 & 302 & 112 & 190 \\
\hline & 8117 & 0 & 0 & 0 \\
\hline & 8118 & 0 & 0 & 0 \\
\hline & Average & 1964.2 & 1927.2 & 53.7 \\
\hline & $\%$ of total no. & & $98.1 \%$ & $1.9 \%$ \\
\hline \multirow[t]{8}{*}{3 months } & 8112 & 1364 & 167 & 1197 \\
\hline & 8108 & 581 & 556 & 25 \\
\hline & 8110 & 367 & 228 & 139 \\
\hline & 8109 & 314 & 231 & 83 \\
\hline & 8111 & 7 & 0 & 7 \\
\hline & 8107 & 0 & 0 & 0 \\
\hline & Average & 438.8 & 197.0 & 241.8 \\
\hline & $\%$ of total no. & & $44.9 \%$ & $55.1 \%$ \\
\hline \multirow[t]{8}{*}{5 months } & 8101 & 2913 & 105 & 2808 \\
\hline & 8105 & 1724 & 0 & 1724 \\
\hline & 8102 & 831 & 0 & 831 \\
\hline & 8103 & 597 & 0 & 597 \\
\hline & 8106 & 582 & 0 & 582 \\
\hline & 8104 & 136 & 0 & 136 \\
\hline & Average & 1130.5 & 17.5 & 1113.0 \\
\hline & $\%$ of total no. & & $1.5 \%$ & $98.50 \%$ \\
\hline
\end{tabular}

${ }^{\text {a }}$ Age of animal when infected with T. solium. All animals were euthanized 3 months p.i.

In a second step, serum samples from infected animals were not pre-treated with trichloro acetic acid (TCA) but used at dilutions of $1 / 10$ and $1 / 100$ in PBS supplemented with $0.5 \%$ Tween 20 (Merck) (PBS-T) to enable a quantitative determination of levels of circulating antigen. Preliminary results (data not shown) demonstrated that omitting the pre-treatment did not affect the test results. The cut-off for the first assay was determined using serum samples of Peruvian pigs known to be negative for porcine cysticercosis. The cut-off value was calculated based on the mean ODs of eight negative reference pig sera (Sokal and Rohlf, 1981). The cysticercosis-negative status of pigs used for negative control samples was determined by (i) detailed carcass dissection and (ii) no antibodies in the EITB for T. solium cysticercosis (Tsang et al., 1989).

In the second assay, a reference standard curve was constructed using serial dilutions of excretory/secretory products of Taenia saginata (TsagES) that were initially used for the development of the monoclonal antibodies (Brandt et al., 1992). The dilutions were made in PBST. A reference curve was included on each ELISA plate. The concentration of circulating antigen was calculated following transformation of the OD value using the equation of the reference curve (quantitative ELISA for circulating antigen). Results from the different groups were compared.

For experimental design 2, the quantitative ELISA for circulating antigen was used with serum samples diluted $1 / 200$.

\subsection{Total Ig detection by ELISA}

The ELISA for detection of serum antibodies was performed using crude metacestode antigen of Taenia crassiceps. Taenia crassiceps cysticerci (Toi strain, kindly provided by Chernin, (1975), and maintained in laboratory mice by two to four passages per year) were harvested from the peritoneal cavity of mice (Swiss A strain) approximately 90 days after experimental infection. The antigen was prepared according to Nunes et al. (2000). This test is based on the cross-reactivity of antigens between Taenia species and also yields a positive reaction in pigs infected with Taenia hydatigena. However, this ELISA was only performed on serum samples from the animals from experimental design 1 that were known to be free of T. hydatigena infection. The ELISA was performed according to Nunes et al. (2000) with some minor modifications: all steps except the incubation of the substrate were done at $37^{\circ} \mathrm{C}$ on a shaking plate, $30 \mathrm{~min}$ incubation for the coating of the antigen, $15 \mathrm{~min}$ for the other incubation steps; blocking was done using PBS, pH 7.2 containing $0.05 \%$ Tween 20 and $2 \%$ newborn calf serum; test serum was diluted 1/200 in blocking fluid; anti-pig IgG (whole molecule) peroxidase conjugate (Sigma A9417) was used at a dilution of $1 / 20,000$ in blocking fluid; OD was read at $492 / 655 \mathrm{~nm}$. All samples were run 2-fold. On each plate two cysticercosis positive pig serum samples and eight negative pig serum samples were run. The cutoff was determined as the mean OD of the eight negative controls plus $3 \mathrm{SD}$. 


\subsection{Data analysis}

The calculation of the standard curve and the statistical analyses were performed using the STATA statistical software, release 7.0 (Stata Corporation 2001, College Station, TX). Correlations between parameters were measured with the Pearson's correlation coefficient for linear correlation on normally distributed data. The confidence level was set at $P<0.05$.

\section{Results}

\subsection{Infection}

The total numbers of cysts as well as the numbers of viable and calcified cysts found at necropsy are shown in Table 1. Both within and between age groups, a large variation in the number of cysts was found in each animal. The total numbers of cysts (numbers of viable cysts) ranged between 0-6246 (0-6210), 0-1364 (0-556) and 582-2913 (0$105)$ in the animals infected at 1,3 and 5 months of age, respectively. At least one cyst was found in four of the six pigs infected at 1 month, in four out of the six pigs infected at 3 months and in all pigs infected at 5 months of age. From the pigs that had at least one parasite, these included viable cysts in only one of the pigs infected at 5 months of age, compared with nine of the 10 pigs infected at earlier age levels $(P<0.05)$. From the total cysts recovered at necropsy, $98 \%$ were degenerated in the animals infected at 5 months of age, compared with $55 \%$ in the animals infected at 3 months of age and only $2.7 \%$ in the animals infected at 1 month of age.

\subsection{Levels of circulating antigen}

The kinetics of the serum antigen levels measured by the standard assay revealed a steep increase of circulating antigen levels in four of the six animals infected at 1 month of age, resulting in a plateau level in the antigen ratio between 1 and 2 weeks p.i., which was maintained until necropsy of the animals (Fig. 1). No circulating antigen was detected in the serum of pigs numbers 8117 and 8118 , infected at 1 month of age.

Four of the six animals infected at 3 months of age had high levels of circulating antigen. The increase in their circulating antigen levels was slower compared with the animals infected at 1 month of age, with a plateau level in the antigen ratio reached 3 weeks p.i.

Antigen levels in animals infected at 5 months of age remained low except in two animals (pigs numbers 8106 and 8101) in which they started to increase 3 weeks p.i. In only one of these two animals was a high antigen ratio measured up to necropsy.

To enable the measurement of a quantitative difference in antigen levels between the different age groups, serum samples obtained at 3,6 and 10 weeks p.i. were diluted $1 / 10$ and $1 / 100$ and used in the quantitative ELISA for circulating antigen. The lowest concentration of detectable antigen was $3.9 \mathrm{ng} / \mathrm{ml}$. The individual calculated values are depicted in Table 2 showing that levels reached were markedly higher in the animals infected at 1 month of age compared with animals infected at 3 and 5 months of age.

\subsection{Total Ig ELISA with T. crassiceps antigen}

The kinetics of the total Ig levels is shown in Fig. 1. Serum antibody levels appeared to follow a reverse kinetics compared with circulating antigen. Antibody titres were highest in the animals infected at 5 months of age, starting to increase as early as 3 weeks p.i. Following a steep increase, the antibody response in these animals declined from 8 weeks p.i. onwards (Fig. 1C). In the animals infected at 1 and 3 months of age (Fig. $1 \mathrm{~A}$ and $\mathrm{B}$, respectively) antibody levels increased slowly and remained substantially lower compared with the older age group. In addition, a decline in antibody titres at the time of slaughter was only observed in Pigs numbers 8110 and 8112 (infected at 3 months of age).

\subsection{Relationship between antigen titres and numbers of cysts}

A total of 98 serum samples obtained from pigs with variable numbers of viable cysts $(=45)$, degenerated cysts only $(=14)$ and no cysts $(=39)$ were tested in duplicate. A serial dilution of TsagES yielded a standard curve that was subsequently used to calculate the concentration of antigen in the serum samples. The relationship between the number of viable cysts and the concentration of circulating antigen is shown in Fig. 2, resulting in a regression line with a Pearson's correlation coefficient of 0.89 $\left(R^{2}=0.81, n=98, P<0.001\right)$.

\section{Discussion}

Experimental infections with $T$. solium in pigs are a prerequisite to improve current understanding of the pathogenesis of the disease (Flisser et al., 2002). Recently, an oral infection model was developed in which piglets of defined age levels (1-, 3- and 5-months-old) are infected with a whole proglottid embedded in a banana ball. Although it does not allow determination of the exact level of infection, the use of a whole proglottid has the advantage that it mimics the natural mode of infection. Animals infected at 1 month of age harboured mainly viable cysts upon oral infection with one proglottid, whereas in piglets infected at 5 months of age most cysts were degenerated. The outcome of the infection in animals infected at 3 months of age was intermediate, with a more equilibrated distribution of viable and degenerated cysts. The current study was carried out to investigate whether this model could also be used to study host-parasite relationships and the mechanisms underlying resistance to infection in more detail. An attempt was made to link the parasitolog- 
$\longrightarrow$ antigen ---- antibody
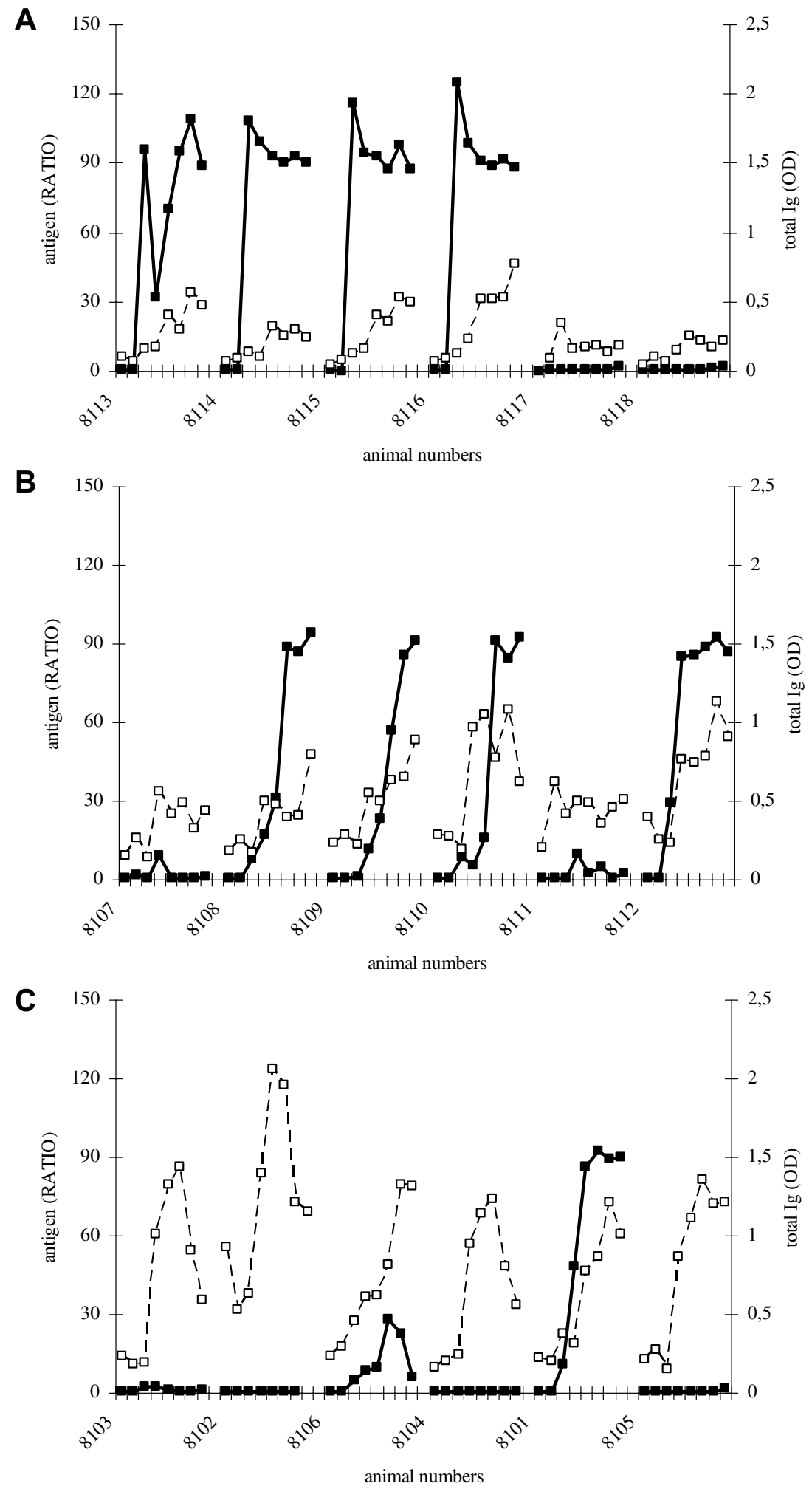

Fig. 1. The serological antigen ( $\square$ ) and antibody $(\square)$ response of 1-month-old (A), 3-months-old (B) and 5-months-old (C) piglets after oral infection with one Taenia solium proglottid. Sera were analysed weekly during the first month p.i., and every 2 weeks thereafter: time points depicted are $0,1,2,3,4,6,8$, 10 and 12 weeks p.i. Antigen levels are expressed as ratio $=\mathrm{OD}$ value of sample/cut-off value (calculated based on the mean OD value (492 nm) of eight non-infected control samples). Antibody values are expressed as OD values as measured with the Taenia crassiceps antibody ELISA.

ical findings to serological data. In a second part of the study, the preliminary observation that levels of circulating antigen seemed to be related to the number of viable cysts was tested more extensively using more animals. 
Table 2

Concentration of circulating antigen $(\mathrm{ng} / \mathrm{mL})$ at 3,6 and 10 weeks p.i. (WPI) in serum of piglets infected with Taenia solium at the age of 1,3 or 5 months

\begin{tabular}{llrcc}
\hline Age $^{\mathrm{a}}$ & Animal no. & 3 WPI & 6 WPI & 10 WPI \\
\hline 1 month & 8115 & 119.32 & 153.39 & 147.7 \\
& 8116 & 13.49 & 127.52 & 132.69 \\
& 8113 & $\leqslant 3.90$ & 15.28 & 87.5 \\
& 8114 & 8.71 & 82.3 & 142.5 \\
& 8117 & $\leqslant 3.90$ & 4.05 & $\leqslant 3.90$ \\
3 months & 8118 & $\leqslant 3.90$ & $\leqslant 3.90$ & $\leqslant 3.90$ \\
& 8112 & $\leqslant 3.90$ & $\leqslant 3.90$ & 13.1 \\
& 8108 & $\leqslant 3.90$ & 6.43 & 40.4 \\
& 8110 & $\leqslant 3.90$ & 4.87 & 13.27 \\
5 months & 8109 & $\leqslant 3.90$ & $\leqslant 3.90$ & 13.67 \\
& 8111 & $\leqslant 3.90$ & $\leqslant 3.90$ & 4.12 \\
& 8107 & $\leqslant 3.90$ & $\leqslant 3.90$ & 4.1 \\
& 8101 & $\leqslant 3.90$ & 21.37 & 79.73 \\
& 8105 & $\leqslant 3.90$ & $\leqslant 3.90$ & 4.07 \\
& 8102 & $\leqslant 3.90$ & $\leqslant 3.90$ & $\leqslant 3.90$ \\
& 8103 & $\leqslant 3.90$ & $\leqslant 3.90$ & 4.07 \\
& 8106 & $\leqslant 3.90$ & $\leqslant 3.90$ & $\leqslant 3.90$ \\
& 8104 & $\leqslant 3.90$ & $\leqslant 3.90$ & $\leqslant 3.90$ \\
\hline
\end{tabular}

${ }^{\text {a }}$ Age of animal when infected with $T$. solium. All animals were euthanised 3 months p.i.

Although detailed carcass dissections at necropsy revealed a high variation in numbers of cysts, the trend was that the number of viable cysts decreased with the age at which the animals were infected. The presence of viable cysts in tissues of infected pigs was monitored by means of an ELISA for the detection of circulating antigen (Brandt et al., 1992). The kinetics of the antigen levels throughout the course of the infection differed markedly between the three age groups of the experimental infection model. In the younger animals a fast increase (starting 1 week p.i.) in titres of circulating antigen was observed in most animals, reaching a plateau as early as 2 weeks p.i.
This demonstrates that antigen can be produced well before cysts are fully developed, which takes approximately 3 months (Pawlowski, 2002). An interesting observation was that animals with viable cysts at necropsy (eight animals) had invariably high antigen levels, whereas animals with no cysts (three animals) or only degenerating cysts (seven animals) had low or nil antigen levels. The transient rise of circulating antigen in pig number 8107 , which was negative at necropsy, probably suggests that the infection was aborted early in its course. The lack of circulating antigen in the other necropsy-negative animals indicates that viable cysts never developed in these animals.

Antigen levels in pigs infected at younger ages not only increased faster but also reached higher levels than in older animals, and were associated with weaker antibody responses. Based on the presence of viable cysts and the higher antigen levels in the younger animals one could hypothesise that cysts circumvent a host immune response in the younger animals, develop faster and remain viable for a longer period of time. In addition, the rapid increase in antibodies in the older animals may be responsible for the abrogation of the development of cysts, which then immediately evolve to a caseous and degenerated status. The classical view of the evolution of cysticercosis lesions assumes that they all pass though the established viable stage, but recently the hypothesis that a substantial proportion of parasites are destroyed in early phases was also put forward to explain the distinct outcome of infection of human NCC (Garcia et al., 2003).

Overall, our observation that higher total Ig titres are present in animals with a higher proportion of degenerated cysts contrasts with other observations showing that antibodies were mostly found in cases with live and involuting parasites, but only very few antibodies were detected in conjunction with degenerated cysts (de Aluja et al., 1996; Flisser et al., 2002). Another striking difference in the

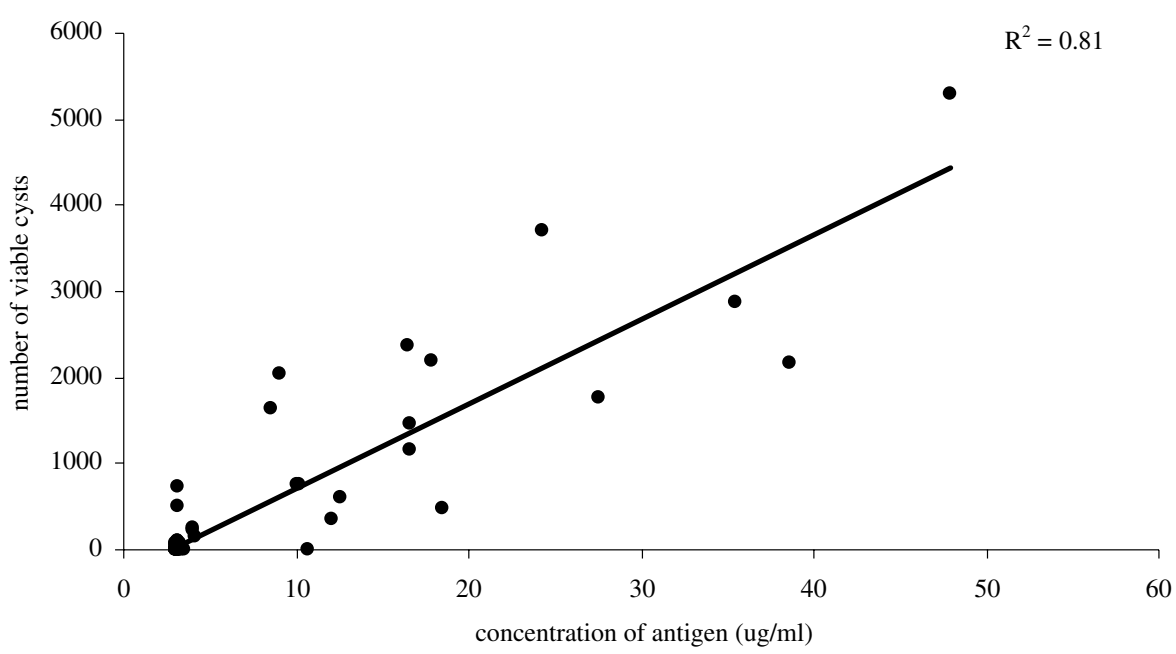

Fig. 2. The correlation between the concentration of circulating antigen and the number of viable cysts in Taenia solium infected animals and non-infected control animals $(n=98)$. Antigen concentrations were calculated following transformation of the OD value using the equation of a reference curve obtained after serial dilution of Taenia saginata excretory/secretory products. 
antibody response was that in all animals infected at 5 months of age, and in two out of the six animals infected at 3 months of age, antibody levels were already decreasing at the time of necropsy ( 84 days p.i.). Upon experimental infection of pigs with 100,000 T. solium eggs de Aluja et al. (1996) also observed a decrease in antibody titres starting from 92 days p.i. However, even at a lower level, antibody titres remained positive up to 280 days p.i. A full comparison between experiments is not feasible as differences may have occurred at the level of the animals (breed), the parasites (difference in infectivity) and infection mode $(100,000$ eggs versus a full proglottid).

The conclusion that the presence of antibodies is influenced by the stage of the parasite is also true for our study, but we would associate higher antibody responses with cyst degeneration. In addition, a role for antibodies is suggested by the rapid increase to high antibody titres associated with an abrogated development of cysts in the older animals. Although evidence for a role of antibodies in inhibition of the development of $T$. solium parasite stages has not yet been reported for porcine cysticercosis, in mice infected with $T$. crassiceps, antibodies were shown to have a crippling effect (Garcia et al., 2001).

In $T$. saginata infection in cattle, the evidence for age resistance has been conflicting (reviewed by Rickard and Williams, 1982). Early reports, using animals that may have been free of prior exposure, demonstrated that although resistance (as related to the ability to become infected and the number of developing cysts) did not develop with age, older animals appeared to have an increased proportion of degenerated cysts (Penfold, 1937; Urquhart, 1961 cited by Rickard and Williams, 1982). This coincides with our findings. Whether the lower numbers of cysts in the older animals are artefacts or reflect the true situation is difficult to interpret as experiments with out-bred animals are inherently exposed to a high amount of variability (Hein and Griebel, 2003) and only a small number of animals were included in this study. Alternatively, the reduction in numbers of cysts in the older animals may have resulted from more effective innate immune responses in these animals. All animals were naïve prior to infection, originating from a cysticercosis-free area, excluding exposure to $T$. solium. This implies an important role for the innate immune response in the initial struggle between the host and the parasite. This is further supported by the abrogation of the viability of cysts at a very early stage of infection, too early to be the result of acquired immunity.

In a second part of the study, we investigated whether the suggested relationship between levels of circulating antigen and numbers of viable cysts, as was observed in the experimental infection model, could be strengthened with the analysis of a higher number of serum samples obtained from a more heterogeneous set of pigs infected with $T$. solium. Despite the variation in ages of animals, in experimental versus natural infections a significant correlation between the number of viable cysts and the concentration of circulating antigen was found. A significant correlation does not necessarily implicate a causal relationship but we may conclude that the variation in antigen concentration in the serum of animals can be partly explained by the number of viable cysts recovered at necropsy. A more detailed analysis revealed that a threshold in the number of cysts is required for finding this relationship (data not shown). In animals with degenerated cysts only, or less than a total number of 100 cysts, the relationship was less consistent. A threshold of 100 cysts was arbitrarily chosen and might need to be refined. The necessity of a threshold can easily be explained by the sensitivity of our assay. Although it proved able to detect one viable cyst (Dorny et al., 2004), up to now, we do not know whether this is entirely reproducible. Maybe the age and development stage of the cysts also play a role in this regard. In addition to the number of viable cysts, the concentration of circulating antigen could be influenced by both hostderived and parasite factors such as the immune status of the host, the location and size of the cysts or genetic differences.

The observation that a relationship exists between the number of cysts and the titre of circulating antigen in pigs is promising in view of the development of an assay to quantify the progress of an active $T$. solium infection. This would be a useful tool in epidemiological studies to assess the infection burden and the risk of transmission of the disease. A recent report showed that antibody titres measured by the FAST-ELISA with sTs18VAR-1 seemed to correlate well with viability of the cysts (Handali et al., 2004). The use of specific antibody-detection assays combined with circulating antigen detection could improve our understanding of this relationship.

\section{Acknowledgments}

Prof. Dr. E. Cox from the Laboratory of Immunology, Faculty of Veterinary Medicine, Ghent University is thanked for helpful discussions. We are grateful to Dr. T. Marcotty for contributing to the statistical analysis.

\section{References}

Brandt, J.R.A., Geerts, S., De Deken, R., Kumar, V., Ceulemans, F., Brijs, L., Falla, N., 1992. A monoclonal antibody-based Elisa for the detection of circulating excretory-secretory antigens in Taenia saginata cysticercosis. Int. J. Parasitol. 22, 471-477.

de Aluja, A.S., Villalobos, A.N.M., Plancarte, A., Rodarte, L.F., Hernandez, M., Sciutto, E., 1996. Experimental Taenia solium cysticercosis in pig: characteristics of the infection and antibody response. Vet. Parasitol. 61, 49-59.

de Aluja, A.S., Villallobos, N.M., Nava, G., Toledo, A., Martinez, J.J., Plancarte, A., Rodarte, L.F., Fragoso, G., Sciutto, E., 2005. Therapeutic capacity of the synthetic-based vaccine against Taenia solium in pigs. Vaccine 23, 4062-4069.

Dorny, P., Vercammen, F., Brandt, J., Vansteenkiste, W., Berkvens, D., Geerts, S., 2000. Sero-epidemiological study of Taenia saginata cysticercosis in Belgian cattle. Vet. Parasitol 88, 43-49. 
Dorny, P., Phiri, I.K., Vercruysse, J., Gabriel, S., Willingham 3rd, A.L., Brandt, J., Victor, B., Speybroeck, N., Berkvens, D., 2004. A Bayesian approach for estimating values for prevalence and diagnostic test characteristics of porcine cysticercosis. Int. J. Parasitol. 34, 569-576.

Flisser, A., Lightowlers, M.W., 2001. Vaccination against Taenia solium cysticercosis. Mem. Inst. Oswaldo Cruz 96, 353-356.

Flisser, A., Correa, D., Evans, C.A.W., 2002. Taenia solium cysticercosis: new and revisited immunological aspects. In: Singh, G., Prabhakar, S. (Eds.), Taenia solium cysticercosis: from basic to clinical science. CAB Int., pp. 5-24.

Flisser, A., Gauci, C.G., Zoli, A., Martinez-Ocana, J., Garza-Rodriguez, A., Dominguez-Alpizar, J.L., Maravilla, P., Rodriguez-Canul, R., Avila, G., Aguilar-Vega, L., Kyngdon, C., Geerts, S., Lightowlers, M.W., 2004. Induction of protection against porcine cysticercosis by vaccination with recombinant oncosphere antigens. Infect. Immun. 72, 5292-5297.

Garcia, G., Sciutto, E., Fragoso, G., Cruz-Revilla, C., Toledo, A., Villalobos, N., Flores, I., Aluja, A., Jose, M.V., Larralde, C., 2001. Inhibitory role of antibodies in the development of Taenia solium and Taenia crassiceps toward reproductive and pathogenic stages. J. Parasitol. 87, 582-586.

Garcia, H.H., Gilman, R., Martinez, M., Tsang, V.C.W., Pilcher, J.B., Herrera, G., Diaz, F., Alvarado, M., Miranda, E., 1993. Cysticercosis as a major cause of epilepsy in Peru. Lancet 341, 197-200.

Garcia, H.H., Gilman, R.H., Tsang, V.C., Gonzales, A.E., 1997. Clinical significance of neurocysticercosis in endemic villages. The cysticercosis working group in Peru. Trans. R. Soc. Trop. Med. Hyg. 91, 176-178.

Garcia, H.H., Del Bruto, O.H., 2000. Taenia solium Taeniasis/Cysticercosis. Infect. Dis. Clin. North Am. 14, 97-120.

Garcia, H.H., Pretell, J., Gilman, R.H., 2002. Neurocysticercosis and the global world. J. Neurol. 249, 1107-1108.

Garcia, H.H., Gonzales, A.E., Gilman, R.H.for the cysticercosis working group in Peru, 2003. Diagnosis, treatment and control of Taenia solium cysticercosis. Curr. Opin. Infect. Dis. 16, 411-419.

Garcia, H.H., Gonzalez, A.E., Del Brutto, O.H., Tsang, V.C., LlanosZavalaga, F., Gonzalvez, G., Romero, J., Gilman, R.H.Cysticercosis working group in Peru, 2007. Strategies for the elimination of taeniasis/cysticercosis. J. Neurol. Sci. 262, 153-157.

Gonzalez, A.E., Garcia, H.H., Gilman, R.H., Gavidia, C.H., Tsang, V.C.W., Bernal, T., Falcon, N., Romero, M., Lopezurbina, M.T., 1996. Effective, single-dose treatment of porcine cysticercosis with oxfendazole. Am. J. Trop. Med. Hyg. 54, 391-394.

Gonzalez, A.E., Falcon, N., Gavidia, C., Garcia, H.H., Tsang, V.C.W., Bernal, T., Romero, M., Gilman, R.H., 1997. Treatment of porcine cysticercosis with oxfendazole: a dose-response trial. Vet. Rec. 141, $420-422$.

Gonzalez, A.E., Falcon, N., Gavidia, C., Garcia, H.H., Tsang, V.C.W., Bernal, T., Romero, M., Gilman, R.H., 1998. Time-response curve of oxfendazole in the treatment of swine cysticercosis. Am. J. Trop. Med. Hyg. 59, 832-836.

Gonzalez, A.E., Gauci, C.G., Barber, D., Gilman, R.H., Tsang, V.C., Garcia, H.H., Verastegui, M., Lightowlers, M.W., 2005. Vaccination of pigs to control human neurocysticercosis. Am. J. Trop. Med. Hyg. $72,837-839$.

Handali, S., Gonzalez, A.E., Hancock, K., Garcia, H.H., Roberts, J.M., Gilman, R.H., Tsang, V.C.W., 2004. Porcine antibody responses to Taenia solium antigens rGp50 and sTs18var1. Am. J. Trop. Med. Hyg. $71,322-326$.

Hein, W.R., Griebel, P.J., 2003. A road less travelled: large animal models in immunological research. Nat. Rev. Immunol. 3, 79-84.

Nascimento, E., Costa, J.O., Guimaraes, M.P., Tavares, C.A., 1995. Effective immune protection of pigs against cysticercosis. Vet. Immunol. Immunopathol. 45, 127-137.

Nunes, C.M., Biondi, G.F., Heinemann, M.B., Richtzenhain, L.J., 2000. Comparative evaluation of an indirect ELISA test for diagnosis of swine cysticercosis employing antigen from Taenia solium and Taenia crassiceps metacestodes. Vet. Parasitol. 93, 135-140.

Pawlowski, Z.S., 2002. Taenia solium: basic biology and transmission in Taenia solium cysticercosis. In: Singh, G., Prabhakar, S. (Eds.), From basic to clinical science. CAB Int., pp. 1-13.

Phiri, I.K., Dorny, P., Gabriel, S., Willingham III, A.L., sikasunge, C., Siziya, S., Vercruysse, J., 2006. Assessment of routine inspection methods for porcine cysticercosis in Zambian village pigs. J. Helminthol. 80, 69-72.

Rickard, M.D., Williams, J.F., 1982. Hydatidosis/cysticercosis: immune mechanisms and immunization against infection. Adv. Parasitol. 21, 229-296.

Sarti, E., Flisser, A., Schantz, P.M., Gleizer, M., Loya, M., Plancarte, A., Avila, G., Allan, J., Craig, P., Bronfman, M., Wijeyaratne, P., 1997. Development and evaluation of a health education intervention against Taenia solium in a rural community in Mexico. Am. J. Trop. Med. Hyg. 56, 127-132.

Schantz, P.M., Sarti, E., Plancarte, A., Wilson, M., Criales, J.L., Roberts, J., Flisser, A., 1994. Community-based epidemiological investigations of cysticercosis due to Taenia solium: comparison of serological screening tests and clinical findings in two populations in Mexico. Clin. Infect. Dis. 18, 879-885.

Schantz, P.M., Wilkins, P.P., Tsang, V.C.W., 1998. Immigrants, imaging, and immunoblots: the emergence of neurocysticercosis as a significant public health problem. In: Scheld, W.M., Craig, W.A., Hughes, J.M. (Eds.), Emerging Infections 2. ASM Press, Washington, DC, pp. 213 242.

Sciutto, E., Aluja, A., Fragoso, G., Rodarte, L.F., Hernandez, M., Villalobos, M.N., Padilla, A., Keilbach, N., Baca, M., Govezensky, T., Diaz, S., Larralde, C., 1995. Immunization of pigs against Taenia solium cysticercosis: factors related to effective protection. Vet. Parasitol. 60, 53-67.

Sokal, R.R., Rohlf, J.F., 1981. Biometry: the principals and practice of statistics in biological research. W.H. Freeman, New York.

Tsang, V.C.W., Brand, J.A., Boyer, A.E., 1989. An enzyme-linked immunoelectrotransfer blot assay and glycoprotein antigens for diagnosing human cysticercosis (Taenia solium). J. Infect. Dis. 159, 50-59. 